\title{
Unfairly Faulty Energy Meter Reading due to Inappropriate Use of the Blondel Theorem
}

\author{
Bas ten Have \\ University of Twente, \\ Enschede, The Netherlands \\ email: bas.tenhave@utwente.nl \\ Niek Moonen \\ University of Twente, \\ Enschede, The Netherlands \\ email: niek.moonen@utwente.nl
}

\author{
Tom Hartman \\ University of Twente, \\ Enschede, The Netherlands \\ email: tom.hartman@utwente.nl \\ Frank Leferink \\ University of Twente, Enschede \\ THALES Nederland B.V., Hengelo \\ The Netherlands \\ email: frank.leferink@utwente.nl
}

\begin{abstract}
Energy measurements are done according to the Blondel theorem, which states that in a system containing $N$ conductors, $\mathrm{N}-1$ current sensing elements are needed to measure the energy. In energy metering for billing purposes at residences only the line current is measured. However, in this paper it is shown experimentally that discrepancies can occur, because current can flow through the protective earth. This occurred in a real one-phase low-voltage distribution system where a residual current device was not functioning properly and a leakage current could flow. This resulted in an unbalance between the line and neutral conductor and thus an inappropriate use of the Blondel theorem.
\end{abstract}

Index Terms-Blondel theorem, Distribution system, Energy measurement, Residual current device, Unbalance

\section{INTRODUCTION}

Measuring the energy in a polyphase system is done according to the Blondel theorem [1]. This theorem states that in a system containing $N$ conductors, $N-1$ current sensing elements are sufficient to measure the electrical energy consumption.

This theorem is used in low-voltage distribution systems when metering electric energy for billing purposes at residential situations [2]. For example in modern static energy meters [3], which were recently investigated because of errors in the energy readings [4].

In such energy meters, used in one-phase systems, the energy is determined using the line-neutral voltage and line current, so only one current sensing element is used. Whereas two elements are required if Blondel is followed correctly, as the system contains the line, neutral and protective earth conductor. The use of fewer elements is done because of economical reasons [2]. This reduction is only valid if the system is balanced, and other parts in the system are working properly, such as a residual current device (RCD), that opens

This project has received funding from the EMPIR programme co-financed by the Participating States and from the European Union's Horizon 2020 research and innovation programme. The results found reflect the author's view only. EURAMET is not responsible for any use that may be made of the information it contains. the mains circuit because of safety issues when a leakage current exists [5]. Similarly, for a three-phase system also the protective earth conductor is not taken into account.

In this paper two case studies are described that show this inappropriate use of the Blondel theorem, which may lead to errors in energy metering.

In the first case study, a consumer in The Netherlands complained about an energy reading of his installed static energy meter that was too high, based on the previous reported static meter errors in [4]. However, it turned out that this was not due to misreadings of the energy meter, as the high energy readings resulted from a short circuit in an oven, resulting in a leakage of $1500 \mathrm{~W}$ through the protective earth. Normally this would result in a trigger of the RCD, however the RCD did not function anymore, due to wear of the device. Therefore, a large protective earth current was flowing causing a difference between the current in the line and neutral conductor and creating a really dangerous situation.

In the second case study it is shown that loads with a non-linear behavior can generate common-mode currents that return via the protective earth. This current has high frequency components, compared to the fundamental frequency, which changes the tripping sensitivity of an RCD [5], and causing the RCD to not trip. As a result a difference between the line and neutral current occurs, such that the energy measurement is non-compliant to Blondel, and could therefore in a extreme case result in major differences in energy measurements. This can become a bigger issue because of the increased use of non-linear electronic devices in low-voltage distribution networks. In general the electronic devices include: switched mode power supplies in computers or televisions, rectifiers in chargers, and lamps [6]. In earlier research conducted electromagnetic interference (EMI) cases have already been described when using non-linear loads [7].

The rest of this paper is organized as follows: In Section II the theory behind the Blondel theorem is described. In Section III the Blondel theorem is explained for a one- 
phase distribution network. Then in Section IV the case study involving a broken RCD is elaborated on. Section V describes the laboratory case study with a non-linear load, resulting in a common-mode current. Finally, in Section VI, a conclusion is made about the correctness of the Blondel theorem for energy measurements.

\section{BLONDEL THEOREM}

In this section the theoretical background behind the Blondel theorem is given, as was published first in [1] and will be summarized hereafter.

A system containing $N$ conductors is considered, at any moment in time the currents and potentials on these conductors are given by $i_{n}$ and $v_{n}$, for all $N$. Then at any moment in time, the algebraic sum of the currents on all the conductors is zero, (1).

$$
\sum_{n=1}^{N} i_{n}=0
$$

The instantaneous power is the current multiplied by the potential delivered to the system, (2).

$$
p=\sum_{n=1}^{N} i_{n} v_{n}
$$

By introducing a common point, $M$, with potential $v_{m}$, the instantaneous power can be calculated as a function of the potential difference between the $N$-th conductor and the common point as seen in (3).

$$
p=\sum_{n=1}^{N} i_{n}\left(v_{n}-v_{m}\right)
$$

Which is proven true since it is know from combining (1) and (2) that (4) holds.

$$
\sum_{n=1}^{N} i_{n} v_{m}=v_{m} \sum_{n=1}^{N} i_{n}=0
$$

Which can then be substituted into (2), resulting in (3). Furthermore, when dealing with alternating polyphase currents, the currents and potentials are periodical functions with period $T$, such that the average power can be written as (5).

$$
P_{m}=\frac{1}{T} \int_{0}^{T} p \mathrm{~d} t=\sum_{n=1}^{N} \frac{1}{T} \int_{0}^{T} i_{n}\left(v_{n}-v_{m}\right) \mathrm{d} t
$$

For practical measurements all of the partial powers can be calculated and added algebraically. In these measurements the common point must be chosen such that the relative values of the potentials and the distribution of the currents does not change. This common point is generally the return conductor of the other $N-1$ conductors in the circuit. Lets assume the $N$-th conductor as common point, then (3) can be rewritten as
(6), which shows that only $N-1$ current sensing elements are needed to measure the energy in polyphase systems.

$$
p=\sum_{n=1}^{N} i_{n}\left(v_{n}-v_{N}\right)=\sum_{n=1}^{N-1} i_{n}\left(v_{n}-v_{N}\right)
$$

\section{ONE-PHASE DISTRIBUTION NETWORK}

In the rest of this paper a one-phase low voltage distribution network is considered. Such a system has three conductors: line, neutral, and protective earth. Fig. 1 shows a schematic of such a system. According to the Blondel theorem, (6), and taking the neutral conductor as the common point, the power is determined using (7).

$$
p=\left(v_{L}-v_{N}\right) i_{L}+\left(v_{P E}-v_{N}\right) i_{P E}
$$

In residential energy metering only the first term involving the line-neutral voltage and line current is taken into account, because it is assumed that the system is earthed and no voltage potential exists between the neutral and protective earth conductor, so no current can flow through the protective earth. However, a voltage drop between the neutral and protective earth conductor does exist, caused by an impedance due to the length of the cable between the residential system and the transformer were the neutral is earthed. This voltage drop will increase when higher impedance loads are connected in between the line and neutral conductor, because then more current is flowing through the neutral conductor. Because of this voltage drop a current can flow in the protective earth, thus making the aforementioned assumption incorrect, and in order to determine the total energy the second term in (7) should also be taken into account.

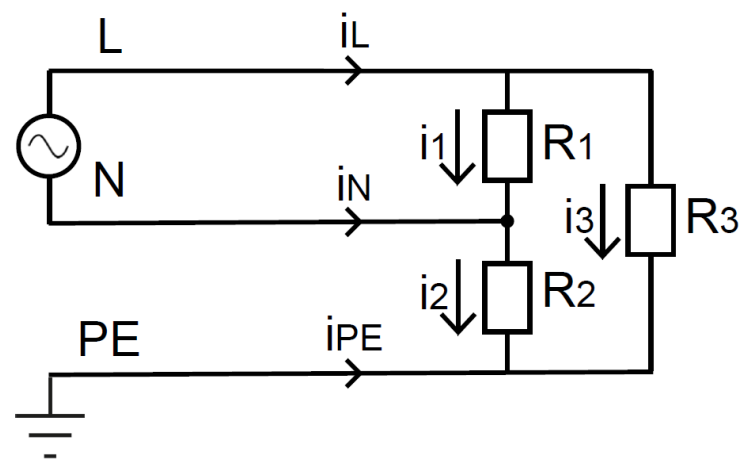

Fig. 1: Schematic of a one-phase distribution system.

Hereafter, two cases are considered in which a protective earth current exists and thus the energy metering is not performed correctly.

\section{CASE STUdy I}

In this section a case study is described in which a broken RCD resulted in a large current flow through the protective earth in a residential situation. This came to light, because the consumer noted an abrupt increase of his energy bill, which could not be explained based on his residential situation. 


\section{A. Monitoring installed static meter}

First of all, the correctness of the readings from the installed static meter were investigated. This was done using a power quality analyzer model PQube Classic from Power Standards Lab, which was installed directly after the static meter. The installed static meter was read out using a pulse counter system using a photo-diode connected to the meter [8]. The meter has a blinking light emitting diode (LED) that gives a blink for every consumed watt-hour of energy. From this measurement it resulted that the readings of the power quality analyzer were in accordance with the static meter readings and therefore questioning these static meter readings was a false assumption.

\section{B. Oven measurement}

Then after switching off (groups of) devices in the residence it was found that the increased energy consumption resulted from the oven in stand-by mode. An energy measurement was done in between the oven and the mains socket in which the oven was plugged in. The line current was measured, using a current probe model TA189 from Pico Technology, and the voltage between the line and neutral conductor was measured using a differential probe model TA043 from the same manufacturer. The transducers were connected to a 5444B Picoscope digitizer with a sampling frequency of $20 \mathrm{MHz}$. A schematic overview of the measurement setup is shown in Fig. 2.

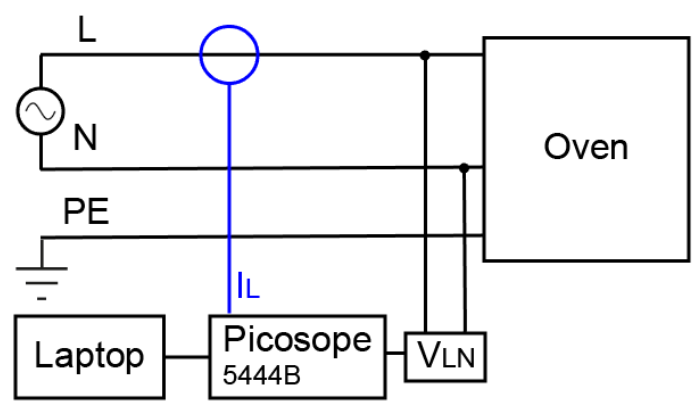

Fig. 2: Schematic overview of the on-site measurement setup for measuring the energy consumption of an oven.

Initially using this measurement no explanation could be found for the high energy reading, because in this case a large consumption of the oven in stand-by mode was perceived. As from Fig. 3 it is clear that a current with a root mean square value of $6 \mathrm{~A}$ was flowing through the line conductor, resulting in a power of $1500 \mathrm{~W}$, without heating any parts of the oven itself.

By coincidence the mains plug of the oven was inverted, swapping the line and neutral conductor, and remarkably the large energy consumption was not measured anymore. From this observation a short circuit was found between the neutral and protective earth conductor, and the unintended path had an impedance of $30 \Omega$. This meant that in the previous case, with the mains plug inverted again, there was a short circuit between the line and the protective earth conductor, causing a large current to run through the protective earth conductor.

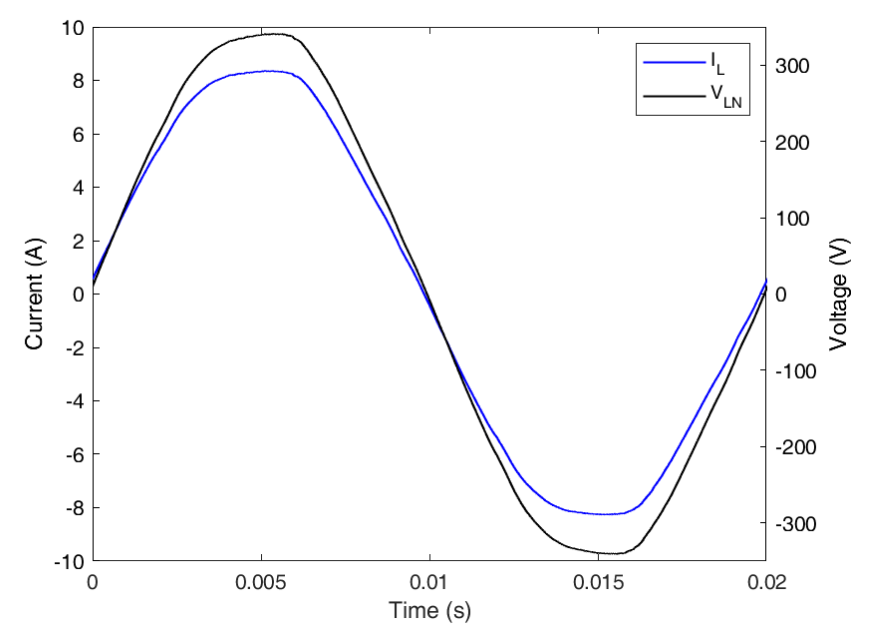

Fig. 3: Line current and voltage between the line and neutral conductor of an oven in stand-by.

This elementary solution was not thought of before, because normally in this situation the RCD would open the mains circuit with such a high current running through the protective earth. However, it turned out that the RCD was broken and did not function anymore, which made it possible for the leakage current to flow. This case study shows that it is not unlikely that such an issue will occur in real household situations.

The measurement results show an unbalance between the line and neutral conductor. Inverting the mains plug of the oven, so swapping the line and neutral conductor, will create a difference in current measurements. This issue is illustrated in Fig. 4 using the electrical circuit and inserting a current element in the line and neutral conductor. In the first situation, the line current element measures the contribution of the load and short circuit, while the neutral current element only measures the current generated by the load, Fig. 4a. In the second situation, the current element in the neutral conductor measures only the current generated by the load, while the neutral current element is also affected by the leakage current, Fig. 4b.

This test case shows that a situation can occur in which the line and neutral currents are unequal, and thus the situation is non-compliant to the Blondel theorem. This has a large effect on the energy measurement used to determine the households consumption for billing purposes.

Another thing that can be learned from this case study is that an RCD may not function because of wear, and one should check the functionality of the RCD regularly to overcome (potential) dangerous situations or over-billing of consumed energy.

\section{CASE STUDY II}

A second case study was performed were a non-linear load is measured under laboratory conditions. As a non-linear load, a commercial of the shelf water pump combined with a 


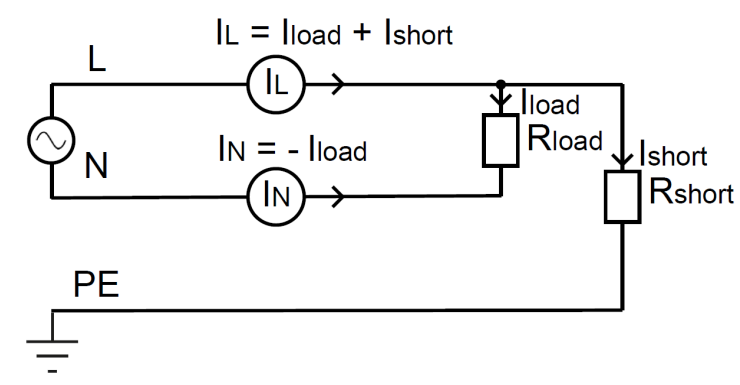

(a) Situation 1

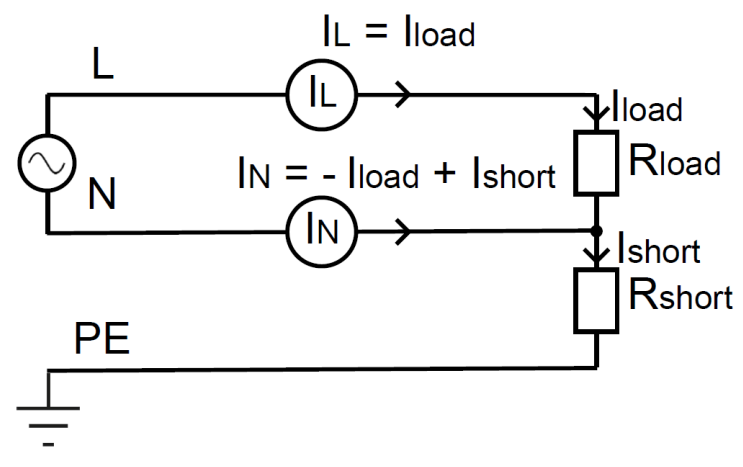

(b) Situation 2

Fig. 4: Resulting electrical circuits when swapping the mains plug of a load with a short circuit.

dimmer was used, this pump already showed large EMI issues on static meters in previous research [9].

\section{A. Measurement setup}

For this study the water pump and dimmer were connected to the mains. The line, neutral and protective earth currents, and the voltage between the line and neutral conductor were measured. This was done using the same transducers and digitizer as previously mentioned. A sampling frequency of $5 \mathrm{MHz}$ was used, and ten cycles at the mains frequency (of $50 \mathrm{~Hz}$ ) were recorded, thus making the measurement interval $200 \mathrm{~ms}$. The measurement setup is shown in Fig. 5.

\section{B. Results}

One period of the recorded waveform is shown in Fig. 6. This measurement shows a current flowing on the protective earth conductor, and an unbalance between the current flowing through the line and neutral conductor. The leakage current shows a damped sine wave with a relatively high frequency compared to the mains frequency, for which a zoomed in plot is shown in Fig. 7.

When it is assumed that no voltage potential exists between the neutral and earth conductor, and measuring the energy according to Blondel the current flowing through either the line or neutral conductor should be measured. As the current on both conductors is measured, the measurement for both conductors can be determined, and these should be identical according to Blondel.

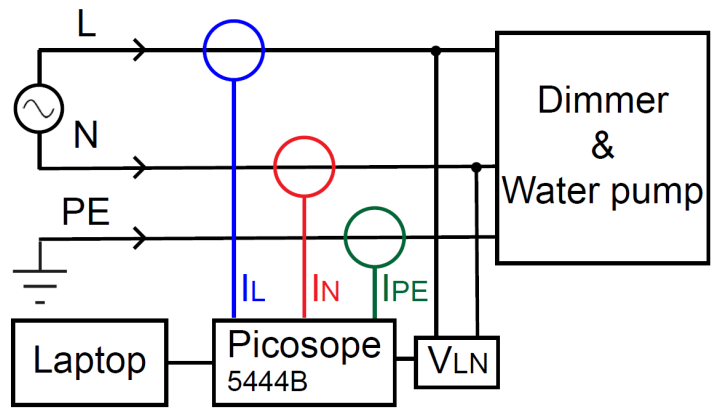

Fig. 5: Schematic overview of the laboratory measurement setup for measuring the voltage and currents from a water pump combined with a dimmer.
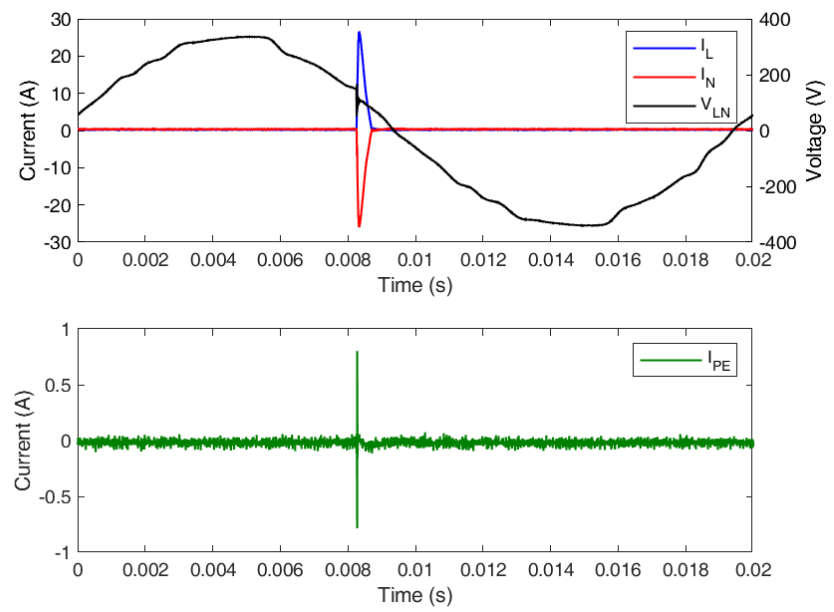

Fig. 6: Voltage and currents for non-linear load, top figure shows the line and neutral current and the voltage, and the bottom figure shows the protective-earth current.

The average power is calculated according to (8) and (9), which agree with the Blondel theorem, where $K$ is the total number of samples.

$$
\begin{gathered}
P_{L}=\frac{1}{K} \sum_{k=1}^{K} I_{L}[k] V_{L N}[k] \\
P_{N}=\frac{1}{K} \sum_{k=1}^{K} I_{N}[k] V_{L N}[k]
\end{gathered}
$$

The calculated power using the line current measurement is $29 \mathrm{~W}$, whereas using the neutral current measurement the calculated power turns out to be $30 \mathrm{~W}$. This shows a difference of $3 \%$ between the two measurements, which can be explained because the current unbalance resulting from a current flowing through the protective earth. This difference is higher than the maximum permissible error limits for electricity meters to be used in Europe with approval of the European Measuring Instrument Directive (MID) according to the European standard 


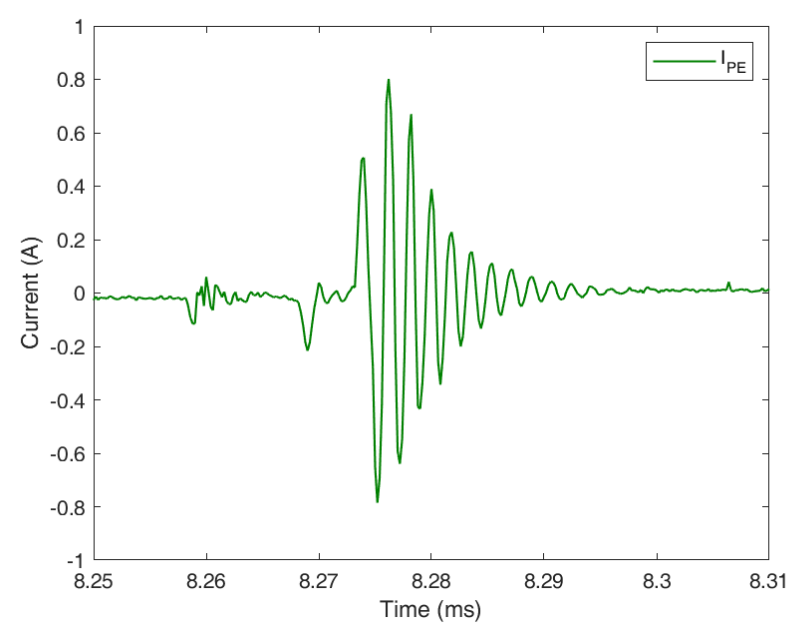

Fig. 7: Zoomed version of the leakage current flowing in the circuit when connecting a non-linear load.

EN 50470-3:2006 [10], which is $2.5 \%$ for Class A electricity meters.

This measurement shows a non-compliance with the Blondel theorem. For electricity metering, e.g. in static meters, this could result in incorrect readings and wrong billing. For example swapping the line and neutral conductor by mirroring the mains plug could be beneficial for consumers. As this case study uses a load with a low energy consumption, the billing effects will not be significantly, however with high power loads that behave non-linear this issue could become problematic.

\section{CONCLUSION}

In this paper it is shown that without measuring the current through both the line and neutral conductor, one cannot determine the current that is flowing through either one of the conductors and thus the current flowing through the protective earth conductor. In energy metering however only the line current is measured. This is only valid if other parts in the system are working properly, for example the RCD that prevents current to flow through the protective earth. Using two case studies showing incomplete electrical energy measurements, the inappropriate use of the Blondel theorem is showed. This could result in wrong energy metering in consumer disputes, and thus incorrect energy billing.

In both case studies, shown in this paper, a current was flowing through the protective earth conductor, creating an unbalance between the current flowing through line and neutral conductor. For the first test case this was due to a broken $\mathrm{RCD}$, and for the second case it occurred because of a nonlinear load. When using the non-linear load a difference of $3 \%$ occurred between the current measurement of the line and neutral conductor.

The issue that is addressed in this paper could easily be avoided by inserting an extra current sensing element when measuring the energy or power of a system.

\section{REFERENCES}

[1] A. Blondel, "Measurement of the energy of polyphase currents." Proceedings of the International Electrical Congress Held in the City of Chicago, August 21st to 25th, 1893, pp. 112-116.

[2] "Progress in the art of metering electric energy: III - Special applications," Electrical Engineering, vol. 60, no. 11, pp. 540-546, 1941.

[3] R. Quijano Cetina, A. J. Roscoe, and P. S. Wright, "Challenges for Smart Electricity Meters due to Dynamic Power Quality Conditions of the Grid: A Review," AMPS 2017 - IEEE International Workshop on Applied Measurements for Power Systems, Proceedings, 2017.

[4] B. Have, T. Hartman, N. Moonen, and F. Leferink, "Inclination of Fast Changing Currents Effect the Readings of Static Energy Meters," 2019 International Symposium on Electromagnetic Compatibility (EMC Europe 2019), pp. 208-213, 2019.

[5] Y. Xiang, V. Cuk, and J. F. Cobben, "Impact of residual harmonic current on operation of residual current devices," 2011 10th International Conference on Environment and Electrical Engineering, EEEIC.EU 2011 - Conference Proceedings, pp. 1-4, 2011.

[6] M. Hossein Pourarab, Morteza; Alishahi, Saeed; Hakkak Sadeghi, "Analysis of harmonic distortion in distribution networks injected by nonlinear loads," 21st International Conference on Electricity Distribution, 2011.

[7] F. Leferink, "Conducted interference, challenges and interference cases," IEEE Electromagnetic Compatibility Magazine, vol. 4, no. 1, pp. 78-85, 2015.

[8] B. Have, C. Keyer, and F. Leferink, "Monitoring of Power Measured by Static Energy Meters for Observing EMI Issues," 2018 International Symposium on Electromagnetic Compatibility (EMC Europe), pp. 903907, 2018.

[9] B. Have, T. Hartman, N. Moonen, C. Keyer, and F. Leferink, "Faulty Readings of Static Energy Meters Caused by Conducted Electromagnetic Interference from a Water Pump," Renewable Energy and Power Quality Journal (RE\&PQJ), 2019.

[10] "EN 50470-3:2006 Electricity metering equipment (a.c.) - Part 3: Particular requirements - Static meters for active energy (class indexes A, B and C), 2006. 